\title{
Mordedura craneofacial por perro
}

\author{
Dr. Carlos Baeza-Herrera, ${ }^{*}$ Dr. Tomás González-Mateos, ${ }^{* *}$ Dra. Adriana Iliana Castillo-Aguirre, ${ }^{* * *}$ \\ Dr. Arturo Hermilo Godoy-Esquivel, ${ }^{* * *}$ Dr. José Antonio Maraboto-Millán ${ }^{* * *}$
}

\section{RESUMEN}

Introducción. El niño es víctima frecuente de mordeduras por perro; las lesiones que causan se deben al componente penetrante del mordisco, y en el caso de una herida craneofacial, la desvitalización, la rotura y machacamiento de los tejidos son la consecuencia más grave y devastadora de la lesión.

El objetivo de este estudio fue analizar las condiciones clínicas predominantes de la herida.

Método. Estudio retrospectivo de 146 pacientes que sufrieron mordedura craneofacial por perro observados entre el 2000 y 2007.

Resultados. Las edades de los pacientes iban de uno a 15 años. Todos los niños fueron hospitalizados y sometidos a reparación primaria de la herida. Doce tuvieron infección local y nueve requirieron la participación del cirujano plástico. Conclusiones. La herida craneofacial por mordedura de perro debe ser suturada por un cirujano, de preferencia pediátrico, con criterios de cirugía estética reconstructiva. Palabras clave: Mordedura por perro, herida craneofacial, cirugía reconstructiva, machacamiento de tejidos.

\section{ABSTRACT}

Introduction. Children are often victim of dog attacks; bite injuries sustained result from the penetrating component of the bite. In craneofacial bites, devitalization, crushing, tearing and avulsion of the facial components are the most serious and devastating consequence of injury.

The purpose of this study was to characterize a group of children suffering craniofacial dog bites and examine the predominant clinical aspect of injury.

Methods. A retrospective review of 146 children with dog bites of the scalp and face was done between the years of 2000 and 2007.

Results. Children's age ranged from 1.0 to 15 years. All patients were hospitalized and underwent primary wound closure; 12 developed local infection and nine required plastic surgery.

Conclusions. Dog bite craniofacial wounds should be treated by pediatric surgeons, with esthetic reconstructive surgery.

Key words. Dog bites, craneo-facial injury, reconstructive surgery, tearing of facial tissue.

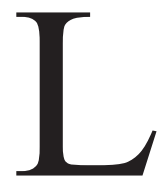

a cercanía con animales ha sido un distintivo que ha caracterizado al ser humano. Desde hace al menos 12 mil años ha habido relación y convivencia con el perro doméstico ${ }^{1}$. En los tiempos modernos, la mayor longevidad de las personas, lo

* Jefe de la División de Cirugía. Hospital Pediátrico Moctezuma. Secretaría de Salud del Distrito Federal. Profesor Titular de Cirugía Pediátrica. Universidad Nacional Autónoma de México.

** Residentes de Cirugía. Hospital Pediátrico Moctezuma.

*** Cirujano Adscrito. Profesor ayudante de Cirugía Pediátrica. Hospital Pediátrico Moctezuma.

Correspondencia: Dr. Carlos Baeza-Herrera. Oriente 158 No. 189

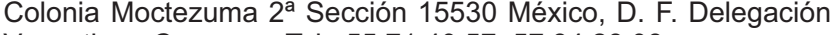
Venustiano Carranza. Tel: 5571405757842808

Correo electrónico: dr.carlosbaeza@yahoo.com.mx

Recibido: septiembre, 2009. Aceptado: marzo, 2010.

Este artículo debe citarse como: Baeza-Herrera C, GonzálezMateos T, Castillo-Aguirre Al y col. Mordedura craneofacial por perro. Acta Pediatr Mex 2010;31(3):89-94.

www.nietoeditores.com.mx poco gregarios que son algunos individuos, el incremento en los crímenes violentos y la gran migración rural a las ciudades ha aumentado de manera notoria la presencia de mascotas en el hogar, sobre todo del perro. En consecuencia ha aumentado el número de lesiones por mordeduras sobre todo en los países donde el control de la población canina es deficiente.

Las heridas de este tipo se producen en distintas regiones incluyendo la cara y el cráneo, aunque preponderan en los miembros pélvicos; también ocurren en las extremidades superiores y en el tronco. La mordedura, una de las más contaminantes, se debe a la acción de incisivos y molares que causan desgarraduras y heridas irregulares que suelen formar colgajos de forma triangular y frecuentemente cursan con isquemia. ${ }^{2}$

El objetivo al revisar este tema fue conocer las características epidemiológicas del fenómeno, analizar la variedad clínica de las lesiones y los resultados de su tratamiento en una unidad hospitalaria como la nuestra, 
de segundo nivel de atención que carece de cirujano plástico.

\section{MATERIAL Y MÉTODO}

Estudio retrospectivo efectuado en la División de Cirugía del Hospital Pediátrico Moctezuma de la Secretaría de Salud del Distrito Federal, entre los años 2000 y 2007 en el que se analizaron los expedientes clínicos de niños menores de 15 años cuyo diagnóstico principal fue mordedura craneofacial por cánido. Se excluyeron del estudio los niños en quienes la mordedura estuvo en otra región. Se analizaron las variables siguientes: a) Del paciente, edad, género, residencia, ubicación y dimensiones de la herida y tiempo de evolución, hora y día de la semana del ataque, relación entre el animal y la víctima y circunstancias en que se desencadenó el ataque. B) Del animal, edad, género, raza y si fueron vacunados contra la rabia. Solamente se analizaron mediciones de tendencia central.

\section{RESULTADOS}

Fueron 91 niños $(62.5 \%)$. Tres pacientes eran menores de un año (2.0\%); entre uno y cuatro años, 82 (56.3\%); entre cinco y diez, 56 pacientes (38.3\%) y más de diez años, cinco (3.4\%).

Todos los pacientes acudieron al Servicio de Urgencias dentro de las primeras 12 horas de la mordedura aunque 23 de ellos habían sido tratados en otro centro hospitalario con curación de la herida. En ningún caso hubo lesión única; 11, además de la lesión craneofacial, tenían mordedura en otro sitio. La cara estuvo involucrada en 55 casos (37.6\%) (Figura 1); el cráneo en 24 (16.4\%) y en el resto fue en ambos sitios. Para describir las heridas de la cara, las dividimos en cuatro variedades, dependiendo del sitio donde se concentró el ataque. Se consideró como central (Figura 2) cuando la herida afectó el triángulo invertido formado por líneas imaginarias que van del punto central del borde inferior del labio inferior al borde supero-externo del arco superciliar de cada lado de la cara; marginal las localizadas en el resto de la cara; especiales como comisura labial y palpebral, (Figura 3 ) orejas, meninges y encéfalo, conductos y glándulas salivales, piso de la boca, arteria y nervio facial; y una última, que es la combinación de las especiales con las centrales. En total observamos 187 heridas craneofaciales y de otras regiones anatómi-

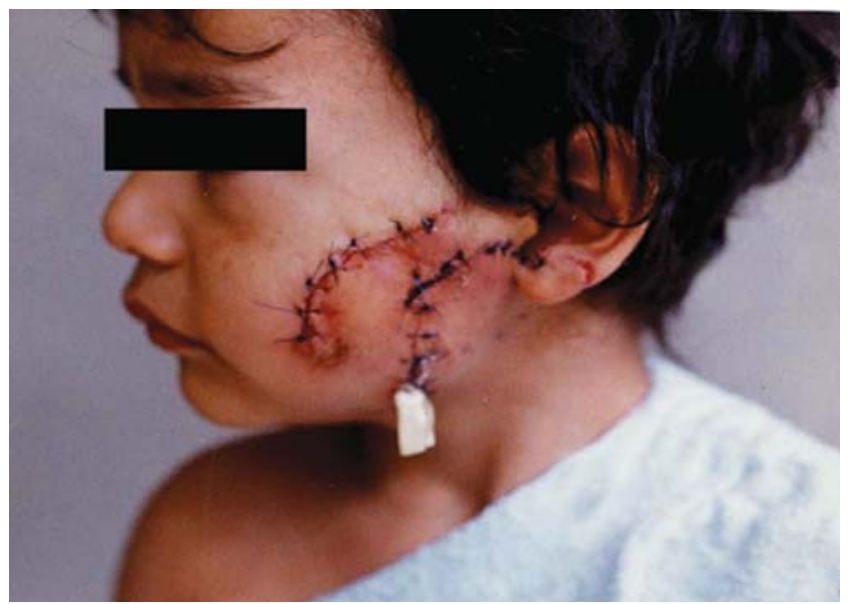

Figura 1. Preescolar con dos heridas en cara. Fue necesario dejar canalizaciones por 24 horas, ya que había tejido subcutáneo necrótico y hemorragia profusa.

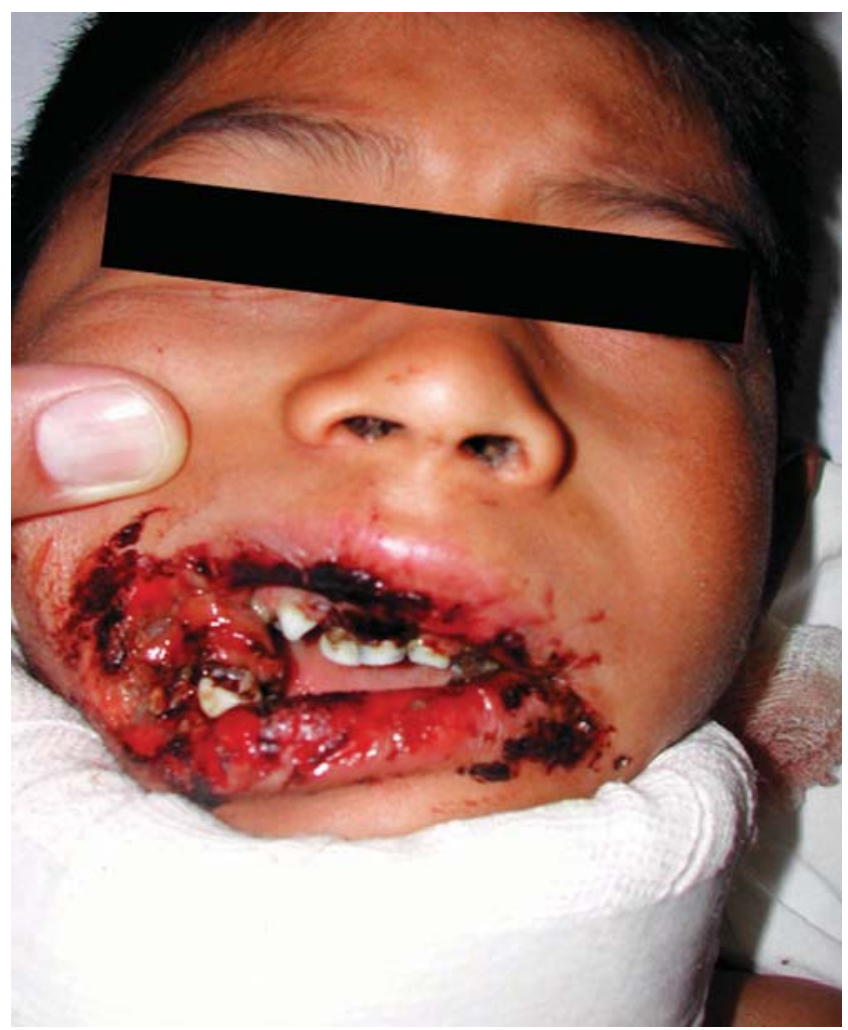

Figura 2. Niño de nueve años mordido por un perro Rottweiler quien le destrozó la boca.

cas; fueron 65 centrales (44.6\%); 41 marginales $(28.0 \%)$; nueve especiales $(6.1 \%)$ y la combinación de lesiones la sufrieron $31(21.3 \%)$. Observamos además como lesión asociada, la fractura de bóveda craneana en dos niños, uno con exposición de las meninges, fractura doble de 


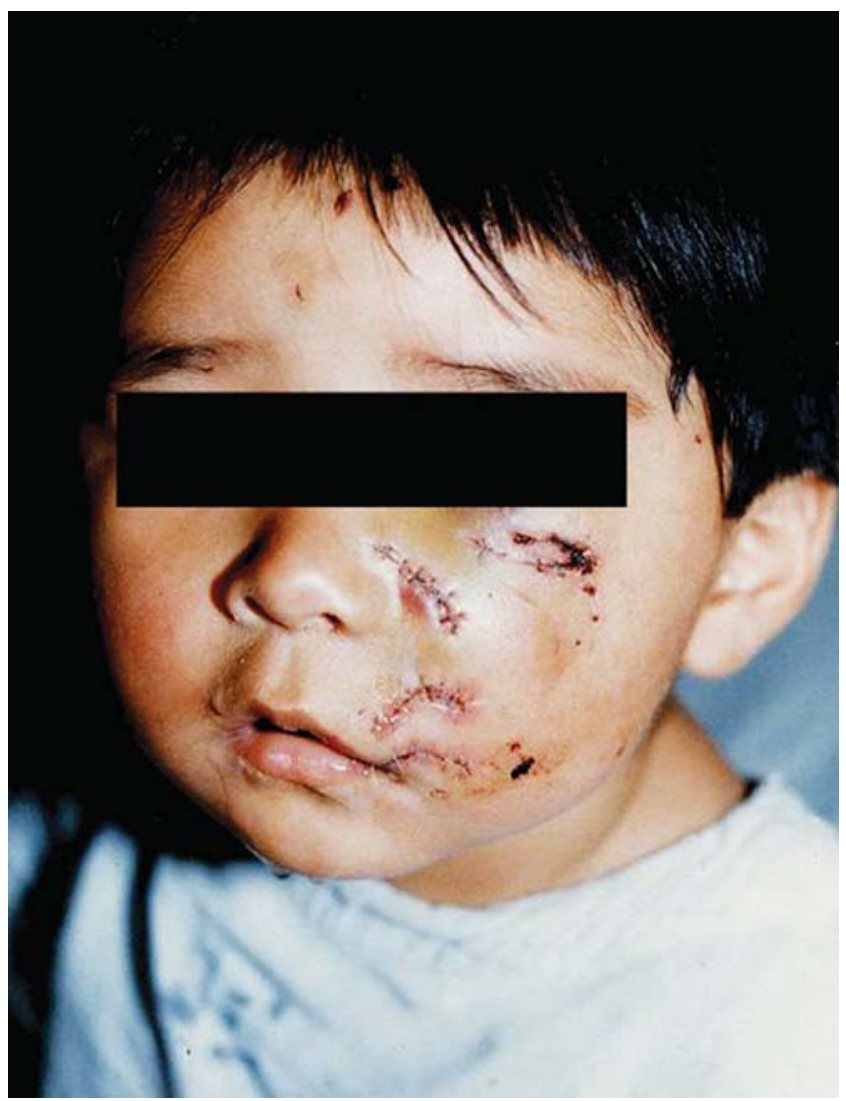

Figura 3. Niño de tres años mordido por el perro de la familia. El niño fue atacado cuando se acercó el perro que estaba comiendo. Son evidentes las lesiones, la del labio superior, lado derecho.

antebrazo en uno y exposición del plexo braquial, arteria y vena humeral en otro. De los 146 pacientes, 13 (8.9\%) tuvieron pérdida de tejido como parte del ala de la nariz, de la oreja o piel cabelluda. Requirieron cirugía plástica en su fase aguda o para corregir algún efecto cosmético, nueve casos $(6.1 \%)$.

La hora del ataque se pudo obtener en 86 pacientes, distribuyéndose de la manera siguiente: entre las seis y las 12 horas, observamos 14 (16.1\%); entre las 12 y las 19 horas 63 (73.5\%); entre las 19 y las seis horas del día siguiente, nueve (10.4\%). Todos fueron operados en el momento de ingreso a la sala de urgencias (Figura 4). La longitud de la herida varió de dos o más lesiones puntiformes, es decir no mayores de $0.5 \mathrm{~cm}$. que no requirieron sutura hasta heridas de 12 centímetros. Doce ( $8.2 \%)$ mostraron datos sugestivos de infección. No hubo decesos. El percance sucedió en el domicilio en 95 niños $(65.0 \%)$ y en otro lugar, en la calle, en una tienda, en un predio, en corredores, etc. en $51(35.0 \%)$.

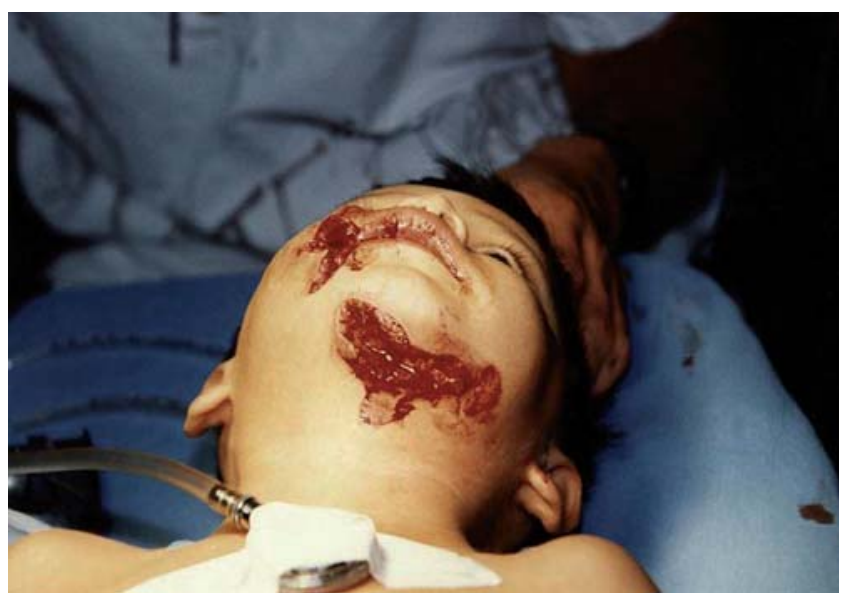

Figura 4. Instantes después de que este niño fue mordido por un perro conocido. Se observan lesiones profundas de cara anterior de cuello y comisura bucal.

El agresor fue perro conocido de la víctima en 95 enfermos (65.0\%); era desconocido en el resto. En todos excepto uno, el ataque fue por un solo animal. Los perros machos agredieron en 99 casos (67.8\%), las hembras en 23 (15.7\%); no se pudo investigar en el resto (16.5\%). La edad del animal sólo se pudo investigar en 96 casos: entre uno y cuatro años de edad, 81 (84.3\%); entre cinco y ocho, $15(15.7 \%)$. En relación a la raza del perro, 37 (25.3\%) eran de raza indeterminada o mestizo; 21 de la familia bull-terrier (14.3\%); pastor alemán 14 (9.5\%) Rottweiler 12 (8.2\%). Los 62 restantes (42.7) estuvieron distribuidos entre alaska-malamut, chow-chow, mastín, akita, Cocker spaniel, Doberman, labrador, sharpei, samoyedo y otras razas no especificadas. La vacunación sólo fue ratificada en 72 animales (49.3\%), no vacunados $23(15.7 \%)$ y en el resto no se conoce.

Circunstancias en que sucedió el percance. En 26 $(17.8 \%)$, sucedió como respuesta a maltrato físico; durante juegos entre el perro y la víctima en 23 (15.7\%); como conducta agresiva repetida en 19 (13.0\%), sin motivo aparente en $16(10.9 \%)$. Otras fueron porque el animal se molestó cuando estaba comiendo, en celo o durmiendo. No encontramos el dato en $52(35.6 \%)$ casos.

\section{DISCUSIÓN}

Probablemente la mordedura de perro de la cara, del cráneo o de ambas, es la lesión más dramática y angustiante para los padres. En los Estados Unidos de Norteamérica, ocurren 300 a 700 ataques de perro por cada 100 mil ha- 
bitantes por año, lo que representa unos 44 mil heridos de cráneo y cara, de las cuales 16 mil son graves ${ }^{2}$. Afecta todas las etnias, pero parece ser más común en latinos que radican en otros países. El perro es conocido de la víctima o de sus padres en casi el $80 \%$ de los casos y el ataque ocurre sobre todo en el interior del hogar ${ }^{3-5}$. El $41 \%$ de los pacientes tiene 18 años de edad o menos ${ }^{6}$ y el $5 \%$ entre cinco y nueve ${ }^{7}$.

Todos los estudios conocidos informan que el problema es más común en los varones, en niños que viven en comunidades urbanas; y en los meses calurosos como primavera y verano y en los fines de semana. El menor número de ataques usualmente acontece en diciembre y enero y el macho es más agresivo con casi el $90 \%$ de los ataques ${ }^{8,9}$.

Los adultos son mordidos habitualmente en las extremidades, pero la frecuencia de mordedura en la cara y cráneo oscila entre el 40 y el $65 \%$ del total y cuando se analizan todos los grupos de edad, mientras es más pequeño es el paciente debe esperarse que el ataque haya sido provocado y la mordedura sea en la cara y el cráneo ${ }^{9,10}$, lo que se debe sin duda alguna a su corta estatura, falta de desarrollo de habilidades motoras, déficit en los mecanismos de defensa, por la empatía que muestran, por la tendencia a acercar la cara en áreas que la mascota percibe como íntima; por su cabeza desproporcionadamente mayor que el resto del cuerpo y porque la cara y cráneo del niño puede ser percibida por el animal como una extensión de su cuerpo o su entorno (territorio) ${ }^{11}$. Según Mcheik et al ${ }^{12}$, las áreas más frecuentemente involucradas son las mejillas, labios, órbita, la frente, mentón, nariz, orejas y la piel cabelluda. Ataques a otros órganos y sistemas como el tórax, abdomen y al sistema nervioso central son afortunadamente las menos; sin embargo, existen y ocasionalmente causan la muerte ${ }^{13,14}$. Las mordeduras de menor gravedad pueden llegan a ser hasta 300 en el cuerpo de un solo niño ${ }^{15}$, pero puede haber daño a estructuras anatómicas como el nervio facial, la laringe, grandes vasos del cuello y la tráquea o determinante de secuelas como epífora, alopecia, ptosis, dificultades para hablar y cicatrices hipertróficas ${ }^{4}$. Cuando la lesión es grave, traduce un patrón que refleja el juego táctico, primitivo y salvaje del perro acosando y destrozando a su presa ${ }^{16,17}$.

Un niño de cuatro años de edad que sufría prolapso rectal y en una ocasión el recto quedó expuesto, colgando del periné; los perros lo atacaron, causándole lesiones colónicas graves. Otro niño de cinco años de edad, al ir a defecar entre unos arbustos, fue atacado por un perro pastor alemán, quien le causó perforación rectal, la que tuvo que tratarse mediante una colostomía ${ }^{15}$.

La gravedad de la lesión por mordedura se debe a que algunas razas como la pit-bull muerden con una fuerza superior a las 1,800 libras por pulgada cuadrada, suficiente para perforar y destruir una lámina metálica. Esta raza no sólo mantiene el mordisco sino que al estabilizarlo, desgarra y tritura los tejidos ${ }^{7}$, dando como resultado desvitalización de grandes colgajos, avulsión y rotura.

El pastor alemán en muchas series es el agresor más común no obstante, se supone que muchos de los perros son catalogados así porque fueron animales grandes y de color oscuro ${ }^{9,18}$.

Un estudio de casos y controles por Gershman et al ${ }^{19}$ halló que las razas que más atacan a humanos son el pastor alemán y el chow-chow; el golden retriever y el chihuahua, son los que menos lesiones ocasionan.

Se han atribuido las causas de la agresividad canina, a socialización inadecuada, al miedo, a trastornos en la concepción de la territorialidad, a una conducta dominante, a predisposición genética, a celos, a maltrato y a sobreprotección. Se supone que en ciertas circunstancias un animal puede malinterpretar gesticulaciones humanas que simulan ser señales de conducta dominante, lo que interpreta como amenaza y despierta la agresividad y casi siempre sucede sobre personas a quien conoce. La agresión cerca de la vivienda del perro, por otro lado, puede tener un fuerte componente de territorialidad. En contraste con lo anterior, los perros callejeros son usualmente cautelosos del ser humano y raras veces son agresivos pero quizá no en comunidades con gran número de perros callejeros 20,11. En México, la mordedura en el $90 \%$ de las veces es consumada por perros de raza inespecífica ${ }^{5}$.

Las heridas faciales, se han clasificado dependiendo de la profundidad en cuatro variedades: I, cuando la lesión no incluye al músculo; II cuando lo incluye; III cuando además hay pérdida de tejido; IVa, lo anterior, más lesión vascular o nerviosa y la IVb, lo anterior, más lesión ósea o pérdida de algún órgano ${ }^{21}$.

En el hocico canino, existen más de 64 especies de bacterias potencialmente patógenas para el ser humano; destacan las aeróbicas como el estreptococo beta hemolítico alfa y beta, estafilococo aureus y epidermidis, 
Pasteurella multocida, E. coli y pseudomona; anaeróbicas como B. fragilis y Prevotella y fisobacterium ${ }^{22}$.

No obstante que la Norma Oficial Mexicana e inclusive libros de texto de cirugía recomendaron no afrontar las heridas de esta naturaleza ${ }^{5,23}$, el tratamiento integral de las heridas por mordedura de perro en cara y cráneo, incluye limpieza y desbridación del tejido necrótico y se recomienda como lo hacemos nosotros, emplear productos antisépticos yodados. La curación incluye revisión de estructuras anatómicas especiales y la remoción de tejido necrótico. Para la reconstrucción de planos profundos, recomendamos el material fino absorbible como vicryl y para la piel, monofilamento fino ${ }^{24,22}$. Ocasionalmente, en una herida antigua por infección activa o hemorragia copiosa, dejamos una canalización, que se retira en las siguientes 24 horas.

Hay estudios en los que no se ha demostrado la utilidad de los antibióticos profilácticos; sin embargo, son necesarios con fines preventivos y curativos. Cuando la herida es extensa y profunda ${ }^{25}$, se recomienda utilizar ampicilina con o sin sulbactam asociada a doxicilina o ceftriaxona ${ }^{23}$.

Las lesiones traumáticas en niños pequeños son previsibles aplicando criterios primarios de prevención como en las mordeduras por perro. Probablemente coadyuvaría la promoción de los cuidados que se les debe a los animales, evitar la "humanización" y el entrenamiento como sustituto de un compañero del niño y hacer entender a la víctima que ante un ataque inminente es recomendable permanecer estático y con los pies juntos y extendidos, boca abajo y cubriendo con las extremidades superiores la cara y cráneo. Otras recomendaciones son: ver directamente a los ojos del animal; evitar abrazar y besar a un perro ya que traduce sumisión y puede confundir al animal y llevarlo a adoptar una conducta agresiva ${ }^{26}$; respetar a los perros especialmente cuando comen, duermen y defecan. No molestarlos ni golpearlos. Jamás jugar con un perro ajeno ${ }^{27}$.

Con propósitos preventivos, los gobiernos municipales deberían implementar programas de control y registro de perros domésticos, en especial de los que son agresivos como el Rottweiler, Doverman, pastor alemán y los bull-terrier para evitar la lesión física y los trastornos secundarios como es el stress postraumático ${ }^{24,28}$.

Este estudio muestra datos que traducen un inconveniente social, pues si bien sólo informa una parte del espectro, si se sumaran todas las mordeduras que acontecen en el país, deben ser cantidad tal, que traducirían como un problema de salud pública que debe ser estudiado y resuelto por las autoridades competentes.

Para tratar cada caso, no es obligado que los hospitales cuenten con cirujano plástico. Según Schulz ${ }^{29}$, la herida por mordedura de perro en cara tiene buen pronóstico estético cuando es reparada por cirujano distinto al plástico y aun sin repararla. En nuestros pacientes operados, dos o más semanas después, los efectos no eran tan desfavorables desde el punto de vista cosmético.

\section{REFERENCIAS}

1. Davis JMS, Valla RF. Evidence for domestication of the dog 12,000 years ago in the Natufian of Israel. Nature. 1978;276:608-10.

2. Karlton AT. The incidente of facial injuries from dog bites. JAMA. 1984;251:3265-7.

3. Navia MJ. Mordedura de perro en cara. Arch Argent Pediatr. 2005;103:396-400.

4. Mitchel BR, Nañez G, Wagner DJ, James K. Dog bites of the scalp, face, and neck in children. Laryngoscope. 2003;113:4925.

5. Martínez MAM. Mordedura de perro en la infancia. Estudio epidemiológico y clínico de 118 casos. Bol Med Hosp Infant Mex. 1998;55:458-62.

6. Borud JL, Friedman WD. Dog bites in the New York city. Plastic Reconstruct Surg. 2000;106:987-90.

7. Tuggle WD, Taylor VD, Stevens JR. Dog bites in children. J Pediatr Surg. 1993;28:912-14.

8. Chun TY, Berkelhamer EJ, Herold ET. Dog bites in children less than 4 years old. Pediatrics. 1982;69:119-20.

9. Méndez MRG, Gómez MT, Somoza IA, Liras JM, Pais PE, Vela DN. Mordedura de perro. Análisis de 654 casos en 10 años. An Esp Pediatr. 2002;56:425-9.

10. Weiss $B H$, Friedman ID, Cohen HJ. Incidence of dog bite injuries treated in emergency departments. JAMA. 1998;279;51-3.

11. Brogan VT, Bratton LS, Dowd D, Hegenbarth AM. Severe dog bites in children. Pediatrics. 1995;96:947-50.

12. Mcheik NJ, Vergnes $P$, Bondonny MJ. Treatment of facial dog bite injuries in children: A retrospective study. J Pediatr Surg. 2000;35:580-3.

13. Sacks JJ, Lockwood R, Hornreich J, Satín WR. Fatal dog attacks. 1989-1994. Pediatrics. 1996;97:891-5.

14. Wilberger EJ, Pang D. Craniocerebral injuries from dog bites. JAMA. 1983;249:2685-8.

15. Wiseman EN, Chochinov H, Fraser V. Major dog attack injuries in children. J Pediatr Surg. 1983;18:533-6.

16. Suárez O, Burgos L, Aguilar R, Luis A, Encinas JL, et al. Reconstrucción quirúrgica de las lesiones graves por mordedura de perro en niños. Cir Pediatr. 2007;20:148-50.

17. Calkins MC, Bensard DD, Partrick AD, Barrer MF. Life-threatening dog attacks: A devastating combination of penetrating and blunt injuries. J Pediatr Surg. 2001;36:1115-7.

18. Schwartzman DS, Pacin BM. Lesiones por mordedura de perro en niños. Arch Argent Pediatr. 2005;103:389-395. 
19. Gersham AK, Sacks J, Wright CJ. Which dogs bite?. A casecontrol study of risk factors. Pediatrics. 1994;93:913-7.

20. Avner RJ, Baker BD. Dog bites in urban children. Pediatrics. 1991;88:55-7.

21. Lackman MG, Draf W, Isselstein G, Tollner U. Surgical treatment of facial dog bite injuries in children. J CraniomaxilloFacial Surg. 1992;20:81-86.

22. Jackson P, Morgan DDS, Haug HR, Murphy TM. Management of facial dog bite injuries. J Oral Maxillofac Surg. 1995;63:43541.

23. Morgan PJ, Haug HR, Murphy TM. Management of facial dog bite injuries. J Oral Maxillofac Surg. 1995;53:435-41.

24. Akhtar N, Smith MJ, McKirdy S, Page RE. Surgical delay in the management of dog bite injuries in children, does it increase the risk of infection? J Plast Reconstruct Aesthet Surg. 2006;59:80-5.

25. Wolff DK. Management of animals bite injuries of the face: Experience with 96 patients. J Oral Maxillofac Surg. 1998;56:838-43.

26. Presutti JR. Prevention and treatment of dog bites. Am Fam Physician. 2001;63:1567-72.

27. Sokol BA, Houser GR. Dog bites: Prevention and treatment. Comments from the surgeon's viewpoint. Clin Pediatr. 1971;10:336-8.

28. Peters V, Sottiaux M, Appelboom J, Kahn A. Posttraumatic stress disorder after dog bites in children. J Pediatr. 2004;144:17-22.

29. Schulz RC. The treatment of dog bite injuries specially of the face. Plast Reconstruct Surg. 1972;48:494-500. 